\title{
Chapter 16 ATP Imaging in Xenopus laevis Oocytes
}

\author{
Takashi W. Ijiri, Jun-ichi Kishikawa, Hiromi Imamura, \\ Yasuhiro Iwao, Ken Yokoyama, and Ken-ichi Sato
}

\begin{abstract}
Adenosine 5' -triphosphate (ATP) is a major energy currency for various chemical reactions in living cells. In spite of its significant roles, the local ATP timecourse in Xenopus oocyte/egg has not been studied fully yet. Therefore, our goal is to understand the molecular mechanisms of maturation and fertilization by analyzing ATP in oocytes and eggs. A fluorescence resonance energy transfer (FRET)based ATP indicator, named ATeam, has been developed and enabled ATP imaging. We are applying this latest experimental technique to observe local ATP in Xenopus oocytes and eggs during maturation and fertilization. First, we used full-grown oocytes to set up the experimental conditions. To observe ATeam fluorescence in Xenopus oocytes, the translucent oocytes were prepared after injecting ATeam protein into the vegetal hemisphere. Then they were observed under fluorescence microscopy, and FRET was measured by analyzing their images using software. ATeam displayed strong FRET with low background. More importantly, FRET signal of ATeam consistently increased in response to the addition of ATP, suggesting that ATeam works in the translucent oocytes. Using this Xenopus ATP imaging system, ATP distribution is investigated not only in oocytes during maturation but also in eggs during fertilization.
\end{abstract}

Keywords ATP imaging • Maturation $\bullet$ Oocyte $\bullet$ Xenopus laevis

\footnotetext{
T.W. Ijiri • J. Kishikawa $\bullet$ K. Yokoyama $(\square) \bullet K$. Sato $(\bowtie)$

Department of Molecular Biosciences, Faculty of Life Sciences, Kyoto Sangyo

University, Kyoto 603-8555, Japan

e-mail: yokoken@cc.kyoto-su.ac.jp; kksato@cc.kyoto-su.ac.jp

H. Imamura

The Hakubi Center for Advanced Research, Kyoto University, Kyoto 606-8302, Japan

Y. Iwao

Department of Applied Molecular Biosciences, Graduate School of Medicine,

Yamaguchi University, Yamaguchi 753-8512, Japan
} 


\subsection{Introduction}

Adenosine 5' -triphosphate (ATP) works as intracellular energy transfer for many reactions of the living body in a wide variety of organisms. For all its importance, ATP distribution in the cells could not be investigated because methods for local ATP detection were lacking. However, Imamura et al. (2009) developed an ATP indicator, named ATeam, that consists of CFP and YFP fused with the $\varepsilon$-subunit of $\mathrm{FoF}_{1}$-ATPase. The $\varepsilon$-subunit changes its conformation into a folded form upon ATP binding, resulting in increase of YFP/CFP ratio under higher ATP concentration. In other words, measurement of its fluorescence resonance energy transfer (FRET) efficiency enables ATP imaging. [For the details of ATeam, please refer the original publication (Imamura et al. 2009).] The number of reports has increased lately about successful ATP observations using ATeam in worms (Kishikawa et al. 2012), in the hepatitis C virus-replicating cells (Ando et al. 2012), in plant cells (Hatsugai et al. 2012), and in flies and worms (Tsuyama et al. 2013) since the first report in HeLa cells (Imamura et al. 2009).

The study of ATP metabolism in the oocyte/egg has been performed mainly in mammals. It was suggested that oxidative phosphorylation in mitochondria is activated during maturation (Brinster 1971; Eppig 1976) and fertilization (Dumollard et al. 2004). In Xenopus oocytes, vast numbers of mitochondria are produced suddenly near the nucleus at early oogenesis; they then localize to the vegetable pole side in the progress of oogenesis. To understand how ATP metabolism occurs in the Xenopus oocyte or egg, observation of local ATP is needed. Therefore, we started ATP imaging in Xenopus oocytes/eggs using ATeam. In this chapter, we show results to set up the ATP imaging system in Xenopus oocytes were successful and discuss its future implications.

\subsection{Methodology}

\subsubsection{Purification of ATeam Protein}

ATeam protein was prepared according to the procedures by Imamura et al. (2009). First, Escherichia coli transformed by ATeam expression plasmid was cultured in LB medium overnight, then the collected bacteria were dissolved in buffer $\mathrm{A}$ [100 $\mathrm{mM} \mathrm{Na}_{3} \mathrm{PO}_{4}$ (pH 8.0), $200 \mathrm{mM} \mathrm{NaCl}$, and $10 \mathrm{mM}$ imidazole]. After treatment by a sonicator, they were applied to a Ni-NTA column and eluted by buffer A with $200 \mathrm{mM}$ imidazole. Furthermore, the concentrated ATeam fraction was purified by gel filtration and replaced in $20 \mathrm{mM}$ Tris- $\mathrm{HCl}(\mathrm{pH} \mathrm{8.0)}$ and $150 \mathrm{mM} \mathrm{NaCl}$. After adding glycerol to purified ATeam protein, it was frozen rapidly in liquid nitrogen and stored at $-80^{\circ} \mathrm{C}$ until use. Protein concentration was determined by the absorption at $515 \mathrm{~nm}$ of YFP. 


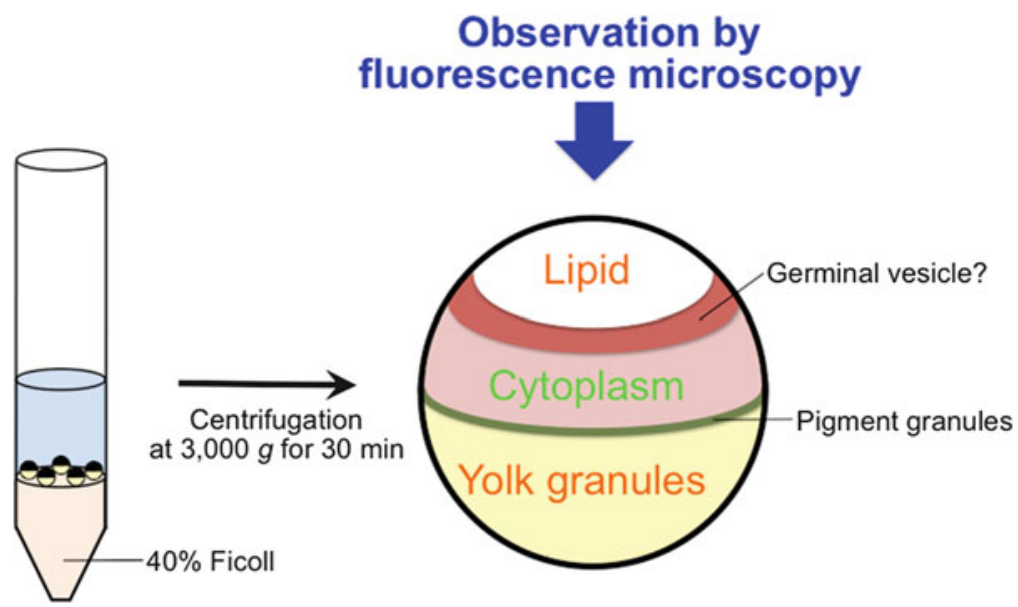

Fig. 16.1 Schematic diagram of the method for translucent oocytes. Left: Oocytes were centrifuged at 3,000 g for $30 \mathrm{~min}$ on a $40 \%$ Ficoll cushion. Right: The oocyte stratified into lipid, a semitransparent layer, clear cytoplasm, pigment granules, and yolk granules from the centripetal to centrifugal side after this treatment

\subsubsection{Preparation of the Translucent Xenopus Oocytes}

Xenopus oocytes and eggs contain pigment and yolk granules that interrupt the observation of fluorescence under microscopy. Therefore, the preparation of translucent oocytes or eggs is needed to observe the FRET signal of ATeam. In this report, we prepared the translucent oocytes using the methods by Iwao et al. (2005). Ovaries were dissected out from adult frogs (Xenopus laevis) and treated with $1 \mathrm{mg}$ / $\mathrm{ml}$ collagenase. After washing, stage VI full-grown oocytes were stored in modified birth solution [MBS: $88 \mathrm{mM} \mathrm{NaCl}, 1 \mathrm{mM} \mathrm{KCl}, 2.4 \mathrm{mM} \mathrm{NaHCO}{ }_{3}, 10 \mathrm{mM}$ HEPES, $\left.0.82 \mathrm{mM} \mathrm{MgSO}_{4}, 0.33 \mathrm{mM} \mathrm{Ca}\left(\mathrm{NO}_{3}\right)_{2}, 0.41 \mathrm{mM} \mathrm{CaCl}_{2}, \mathrm{pH} 7.6\right]$ with $0.01 \%$ chloramphenicol. In advance, ATeam protein (23 or $46 \mathrm{nl}$, corresponding to 0.1 or 0.2 pmol, respectively) was injected into vegetal hemisphere of the oocytes. The oocytes were put onto $40 \%$ Ficoll in MBS, then centrifuged at 3,000 $\mathrm{g}$ for $30 \mathrm{~min}$ (Fig. 16.1). After this treatment, lipids form a cap-like structure on the top of the oocytes; germinal vesicle may surround this lipid layer, and translucent cytoplasm occupies the remaining area in the animal hemisphere, while pigment granules set on the equator and yolk granules consists in whole vegetal hemisphere (Fig. 16.1). These translucent oocytes were put in MBS until observation.

\subsubsection{Observation Under Microscopy and Image Analysis}

The translucent oocytes were observed under fluorescence microscopy (Zeiss, Axioplan2) with the filters D480/30 for CFP and D535/40 for YFP (Photometrics, DualView2). The fluorescence images were taken by a digital camera (Hamamatsu, 

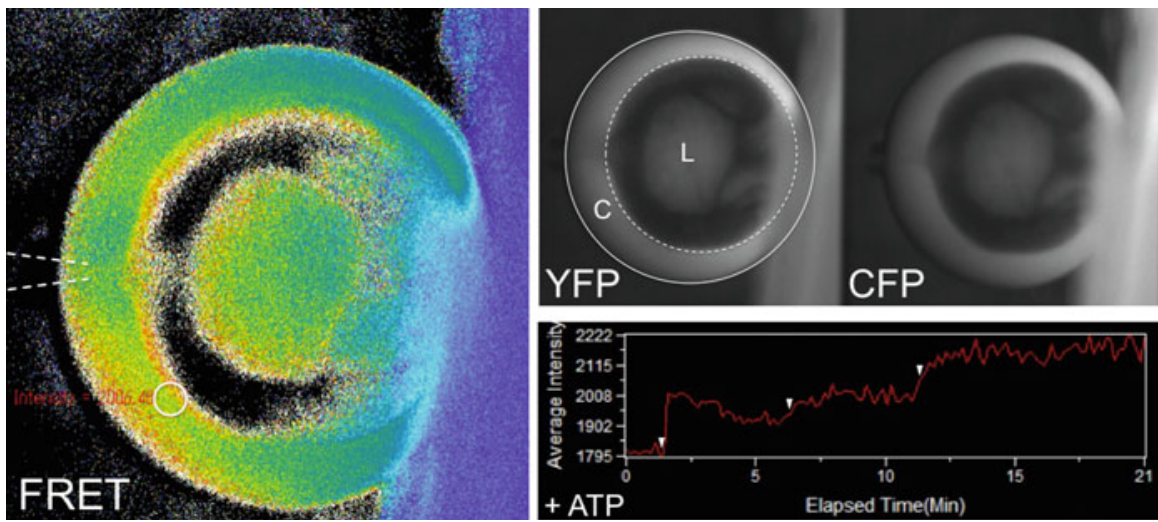

Fig. 16.2 Monitoring of ATeam in Xenopus oocytes. FRET signal of ATeam increased after each of three injections of ATP. Left: The white circle in the FRET image is the spot where average intensity was measured for the line graph. The two white dotted lines indicate a glass needle injected into translucent cytoplasm. Right upper: Each image of ATeam YFP and CFP. $L$ lipid layer, $C$ translucent cytoplasm. Right bottom: White heads in the line graph show the times when ATP was injected. Note: Viewing angle by fluorescence microscopy was from animal pole to vegetal pole. The diameter of the Xenopus oocyte is $1.2-1.3 \mathrm{~mm}$

ORCA-Flash2.8) every $10 \mathrm{~s}$. The fluorescence intensity was measured and then FRET signal of ATeam (YFP/CFP) was calculated using MetaMorph software (Molecular Devices). The results were shown as average intensity (Fig. 16.2), which is 1,000 fold of the FRET value. For the example in Fig. 16.2, 0.2 pmol ATeam protein was injected into an oocyte before treatment for translucent oocytes, then $9.2 \mathrm{nl} 16.5 \mathrm{mM}$ ATP solution was injected three times into an oocyte through a glass needle, which was inserted into the translucent cytoplasm beforehand.

\subsection{Injected ATeam Protein Works in Xenopus Oocytes}

Our preliminary trial using robust ATeam protein resulted in bright fluorescence over all the translucent cytoplasm of unfertilized egg without any background (Ijiri, Kishikawa, Imamura, Iwao, Yokoyama, and Sato, unpublished results). Therefore, the next trial should be performed with minimal ATeam amount to detect accurate ATP distribution in the Xenopus oocyte or egg. This time we used full-grown oocytes for long-term observation because they are easily handed compared to an unfertilized egg; that is, the oocytes have moderate strength and are not activated by stimuli such as injection. After several trials, we decided that $0.1-0.2$ pmol of protein injection per oocyte is suitable for the Xenopus ATP imaging system. In this study, we used modified ATeam, which is optimized for the lower temperature of most model animals (20-25 $\left.{ }^{\circ} \mathrm{C}\right)$ (Tsuyama et al. 2013). Under these experimental considerations, our strategy worked well, and either 0.1 or 0.2 pmol of ATeam protein also produced 
similar results. First, we could observe a strong FRET signal of ATeam with low background over all the translucent cytoplasm in the edge of an oocyte, although a lipid layer blocked in the central part (Fig. 16.2). Then, the FRET did not decrease markedly during the observation time: at least $1 \mathrm{~h}$ (data not shown). The most important thing to note here is that ATeam reacted with the increase of ATP concentration during observation. In this test to evaluate the imaging system, ATP was injected into an oocyte three times between moderate intervals, resulting in constant increase of FRET after each of the three injections (Fig. 16.2). These data suggest that ATeam works accurately in Xenopus translucent oocytes.

\subsection{Conclusions and Future Directions}

Here, we have succeeded in observing the FRET signal of ATeam using translucent oocytes in Xenopus. In most cases, ATeam mRNA was injected into the subject of the research, whereas ATeam protein was used in our Xenopus system. As far as we know, this is the first report about ATP imaging in living Xenopus oocytes. In our methodology, oocytes have to be treated by centrifugation on Ficoll cushion to prepare the translucent cytoplasm; however, this treatment does not disrupt the cleavage of embryos, at least until blastula stage (Iwao et al. 2005). Therefore, for further investigation, we will observe the time-course of local ATP distribution in the oocytes after adding progesterone to detect the difference during oocyte maturation. We will also apply this imaging system with unfertilized eggs to observe local ATP in Xenopus eggs at fertilization. To understand the biological significance of ATP distribution in oocytes and eggs, it is needed to confirm whether ATP localizes with mitochondria using a mitochondrion-selective proves. It is also important to examine the correlation between ATP and $\mathrm{Ca}^{2+}$ with calcium indicators during maturation as well as during fertilization. Another approach will use inhibitors for glycolysis, 2-deoxyglucose, and for oxidative phosphorylation, $\mathrm{KCN}$ and oligomycin $\mathrm{A}$, to define whether ATP is produced only by oxidative phosphorylation in oocytes during maturation and in eggs during fertilization. Our future knowledge from this study can contribute to understanding the fundamental mechanisms of ATP metabolism in vertebrate oocytes and eggs.

Open Access: This article is distributed under the terms of the Creative Commons Attribution Noncommercial License which permits any noncommercial use, distribution, and reproduction in any medium, provided the original author(s) and source are credited.

\section{References}

Ando T, Imamura H, Suzuki R et al (2012) Visualization and measurement of ATP levels in living cells replicating hepatitis C virus genome RNA. PLoS Pathog 8(3):e1002561 
Brinster RL (1971) Oxidation of pyruvate and glucose by oocytes of the mouse and rhesus monkey. J Reprod Fertil 24(2):187-191

Dumollard R, Marangos P, Fitzharris G et al (2004) Sperm-triggered $\left[\mathrm{Ca}^{2+}\right]$ oscillations and $\mathrm{Ca}^{2+}$ homeostasis in the mouse egg have an absolute requirement for mitochondrial ATP production. Development (Camb) 131(13):3057-3067

Eppig JJ (1976) Analysis of mouse oogenesis in vitro. Oocyte isolation and the utilization of exogenous energy sources by growing oocytes. J Exp Zool 198(3):375-382

Hatsugai N, Perez Koldenkova V, Imamura H et al (2012) Changes in cytosolic ATP levels and intracellular morphology during bacteria-induced hypersensitive cell death as revealed by realtime fluorescence microscopy imaging. Plant Cell Physiol 53(10):1768-1775

Imamura H, Nhat KP, Togawa $\mathrm{H}$ et al (2009) Visualization of ATP levels inside single living cells with fluorescence resonance energy transfer-based genetically encoded indicators. Proc Natl Acad Sci USA 106(37):15651-15656

Iwao Y, Uchida Y, Ueno S et al (2005) Midblastula transition (MBT) of the cell cycles in the yolk and pigment granule-free translucent blastomeres obtained from centrifuged Xenopus embryos. Dev Growth Differ 47(5):283-294

Kishikawa J, Fujikawa M, Imamura $\mathrm{H}$ et al (2012) MRT letter: expression of ATP sensor protein in Caenorhabditis elegans. Microsc Res Tech 75(1):15-19

Tsuyama T, Kishikawa JI, Han YW et al (2013) In vivo fluorescent ATP imaging of Drosophila melanogaster and Caenorhabditis elegans by using a genetically encoded fluorescent ATP biosensor optimized for low temperatures. Anal Chem 85(16): 7889-7896 